\title{
LA BORROSA DISTINCIÓN RIESGO-INCERTIDUMBRE
}

\author{
Jlax Glillermo Rivera Berrio ${ }^{1}$
}

\section{Resumen}

Este artículo hace parte de una investigación más amplia en torno a los problemas asociados al riesgo. Los resultados de la investigación presentados se enmarcan en el problema de la polisemia presente en las palabras que representan conceptos como incertidumbre, riesgo y probabilidad.

Se presentan tres posturas que hacen de la distinción riesgoincertidumbre un problema duro de resolver. Una primera postura es no distinguir entre riesgo e incertidumbre, propio de los que he denominado científicos sociales; otra, es definir el riesgo como una categoría de incertidumbre en la cual se habla de grados de incertidumbre; y la tercera postura, que es la que defiendo, es discernir entre incertidumbre y riesgo. Por contraste con la segunda postura, propongo un concepto de riesgo que se construye desde el conocimiento por grado, es decir, el riesgo como grado de conocimiento. $\mathrm{Al}$ estar asociado el riesgo al concepto de probabilidad, mostraré un concepto de riesgo más cercano a los denominados grados de creencia, no como probabilidades, sino como "posibilidades"2.

: Ingeniero Civil y especialista en estructuras de la Universidad Nacional de Colombia. Estudiante de Doctorado Estudios en Ciencia y Tecnología, y Gestión de Innovación Tecnológica en la Universidad del País Vasco-UPV/EHU. Miembro del grupo de investigación de CTS del ITM. Jefe Oficina de Planeación del ITM.

E-mail: juanrivera@itm.edu.co

2 Dubois (2006, pág. 48) establece cuatro ideas a las que puede conducir la palabra posibilidad: viabilidad o factibilidad, como en el caso de la viabilidad de proyectos para la solución de problemas; plausibilidad, aceptabilidad o credibilidad, referido al grado de confianza de que un evento suceda; consistencia con la información disponible, como la proposición que no contradice dicha información; una última

Fecha de recepciòn: 6 de noviembre de 2007 Fecha de aceptación: 21 de diclembre cie 2007

Revista TECNO-LóGICAS No. 19. Diciembre de 2007 


\section{Palabras clave}

Riesgo, Incertidumbre, Probabilidad.

\section{Abstract}

This paper is part of a wider research concerning problems associated to risk. The research results are framed within the problem of the polysemy of words representing concepts such as uncertainty, risk and probability.

Three approaches that turn the risk-uncertainty distinction hard to be resolved are presented. The firs approach is the lack of differentiation between risk and uncertainty which is a characteristic of those here called social scientists. The second one is defining risk as a category of uncertainty and which proposes several degrees of uncertainty. And the third approach, which is the one supported here, is the discrimination between uncertainty and risk. Unlike the second approach, we propose a concept of risk that is built from the risk as a knowledge degree. As the risk is associated with the concept of probability, a concept of risk closer to the so-called belief degrees will be shown, not as probabilities but as "likelihoods".

\section{Key words}

Risk, Uncertainty, Probability.

idea es de tipo ético y referida a las proposiciones que son posibles dentro de la legalidad o la eticidad. Si bien, Dubois defiende la interpretación epistémica de consistencia con la información, mi propuesta está enfocada a la posibilidad como credibilidad, en el contexto de los grados de creencia. 


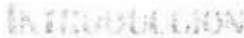

Si diéramos una lectura desde diferentes áreas del conocimiento a algunos textos que abordan el concepto de incertidumbre, podrímos evidenciar que, al igual que en el riesgo, abundan las acepciones que confunden o no permiten establecer un concepto común. Hay propuestas que hablan de incertidumbres por grados (corviente fuertc), hay otras que ven la incertidumbre y el riesgo cemo el reflejo especular de la ignorancia o el desconocimiento (comime débil) '. En los textos de la estadística o de la economía, la incertidumbre es modelada y las ecuaciones resultantes se constituyen, en algunos casos, en herramientas de predicción, no de un futuro escenario, sino de un posible futuro escenario. Desde las ciencias sociales, sin embargo, la confusión reina. La incertidumbre y el riesgo parecen ser una misma cosa, mezclándose 5 confundiéndose en una compleja red de conceptos. El riesgo es representado o interpretado a través de cualidades que le dan el miricter de concepto no consensuado. "Riesgo cuantificable", "riesgo whjetivo", "riesgo subjetivo", "riesgo real", "riesgo global", "riesgo invisible" y "riesgo inductivo", son sólo algunas de las expresiones utilizadas entre una y otra acepción del riesgo ${ }^{4}$. Cada una de ellas

3. Sólo para efectos de este trabajo he clasificado las diferentes propuestas que gradan lit incertidumbre como "corriente fuerte" a aquellas que presentan argumentos para su defensa, ver por ejemplo Wynne [1992], López-Cerezo y Luján (2000), Ravetz (1997), Funtowicz y Ravetz (2001). En contraposición, el riesgo como similitud a la incertidumbre es una propuesta que no ofrece argumentos suficientes para creer en ella. La propuesta débil no hace distinción entre riesgo e incertidumbre, sin embargo, no justifica esta falta de distinción, ver por ejemplo en forma explícita a Rosa (1998) o a Beck (1998 (1986)) implícitamente al referirse a los riesgos invisibles o desconocidos.

" La expresión "riesgo inductivo" es utilizada por Hempel (1965) en su análisis del concepto de probabilidad. Para Hempel el riesgo inductivo está referido a la posibilidad de que una hipótesis falsa sea aceptada como verdad, o que una hipótesis verdadera sea rechazada como falsa. En Hempel (1979 (1966)) no se hace referencia explícita al riesgo inductivo, la aceptación o rechazo de las hipótesis probabilísticas son presentadas más como un error que como un riesgo. Un ejemplo interesante que describe este tipo de riesgo es el siguiente: "Así, si la hipótesis se refiere a la probable efectividad y seguridad de una nueva vacuna, entonces la decisión..." (Hempel, 
surge como resultado del contexto en el cual se estudia el riesgo. Si el riesgo es fruto del análisis de las probabilidades por parte de los expertos, nace el "riesgo objetivo" como contraposición al "riesgo subjetivo". En el contexto del riesgo objetivo, ante la presencia de la incertidumbre, se suele hablar de probabilidades objetivas y probabilidades subjetivas. Riesgo, incertidumbre y probabilidad son conceptos que han sido centro de debates en varias áreas del conocimiento. Sin embargo, es la incertidumbre la que ha generado mayor confusión en estos conceptos. La incertidumbre es fuente inagotable de más y más incertidumbres, de confusiones, de falta de consenso. Es la antítesis del conocimiento. ¿En qué lugar debemos ubicar el riesgo? Mi respuesta, la cual defenderé en este apartado, es la de un riesgo en las regiones del conocimiento. Un concepto de riesgo diferenciado claramente del concepto de incertidumbre.

Es posible que esta propuesta de distinción entre riesgo e incertidumbre sea severamente criticada, con justificadas razones. Justificadas desde el contexto en el que se entiende la incertidumbre o el riesgo (corrientes fuerte o débil). Es posible, también, que en el fondo discutamos sobre lo que estamos de acuerdo. Mi propósito al tratar de establecer esta distinción nace de mi convicción de que la falta de consenso sobre el riesgo tiene sus raíces en otros conceptos (la incertidumbre es uno de ellos), que le son inherentes y que, para lograr un consenso (tarea nada fácil) es importante dejar claro qué entendemos por uno u otro concepto. Situación que a su vez me permitirá elaborar mi propia red de conceptos, a partir de la cual defenderé la necesaria distinción riesgo-incertidumbre ${ }^{5}$.

1979 (1966), pág. 101). Este tipo de decisiones se toman bajo riesgo, si la vacuna es efectiva y se rechaza o si no es efectiva y se emplea con serias consecuencias como resultado, es un riesgo. El calificativo de riesgo inductivo obedece a la experiencia previa para la toma de decisiones. Una frecuencia alta de resultados positivos al aplicar la vacuna en una amplia muestra permite, por inducción, creer que será efectiva en el total de la población. Por su parte, Douglas (2000) define este riesgo como aquel cuyas consecuencias o valores son "no epistémicas".

5 Podrían criticarme por iniciar una discusión más de tipo semántico, cosa que es cierta, pero acaso, ¿las representaciones del riesgo deben eludir la semántica para el logro de sus propósitos? o quizá, ¿no debiéramos criticar severamente a quien 


\section{El RIESGO COMO INCERTIDUMBRE. LA CORRIENTE DEBUL}

El riesgo es incertidumbre. La incertidumbre es riesgo. Esta reflexividad entre los dos conceptos coadyuva a afianzar la "no distinción" de riesgo-incertidumbre. Beck (1998 (1986)) es quizá el que más confusión ha generado al afirmar que en la ignorancia de los riesgos no perceptibles, crecen y prosperan los riesgos. Estos riesgos, dice Beck, se hacen globales en la medida que no conocen fronteras, son universalizados por el aire, el viento, el agua y la cadena alimenticia. (Beck, 1998 (1986), págs. 50-51). ¿Qué quiere decir Beck con ignorancia de los riesgos? Riesgos ignorados son riesgos inexistentes, el riesgo es conocido para ser riesgo ¿cómo podría existir el riesgo de ser invadidos por seres que no conozco de un planeta que no conozco? Beck, entonces, se refiere a incertidumbres epistémicas. De ser así, su sociedad del riesgo es una sociedad de la incertidumbre. Una sociedad con un grado de conocimiento bajo o nulo en lo que respecta a los nuevos riesgos (cambio climático dos décadas atrás, por ejemplo), es una sociedad de incertidumbres.

Dada la poca profundización en el concepto de incertidumbre, por parte de esta corriente, mi análisis se centrará en la corriente fuerte. Es en la corriente fuerte donde mayor debate se ha dado, permitiendo confrontar y criticar lo que por incertidumbre se entiende.

\section{GRADOS DE INCERTIDUMBRE. LA CORRIENTE FUERTE}

Para esta corriente el riesgo es incertidumbre, pero en grado. Esta propuesta, en principio, pareciera ser una posible fuente de distinción. La incertidumbre es el complemento (en términos de la lógica) del grado de conocimiento sobre un evento natural o artifi-

escribe "anti-sísmico" en lugar de "sismo resistente"? Por mi parte, me opongo a ello y, pondré todo mi esfuerzo por demostrar que en estas aparentes nimiedades se encuentra un trasfondo que no ha permitido un lenguaje común en torno al riesgo. 
cial. Es decir, es lo que ignoramos para que nuestro conocimiento sea completo. En esta primera descripción de la corriente fuerte de la incertidumbre se presentan los primeros interrogantes: iexiste conocimiento total o acabado?, ¿cuál es ese complemento a nuestro grado de conocimiento? Si como respuesta a la segunda pregunta obtuviéramos: "aquello que ignoramos", entonces los interrogantes no cesarían: ¿cómo medir la ignorancia?, ¿cómo saber cuál es el grado de incertidumbre?, ¿cuál es el referente para medir el grado de incertidumbre? Estas preguntas, desde la descripción inicial de esta corriente, son lógicamente formuladas y al parecer sin respuesta positiva. No podemos predecir en la ignorancia y menos tomar decisiones en lo incierto, en lo desconocido. La incertidumbre es duda. En la incertidumbre no existen suficientes razones para creer o para tomar decisiones.

Es un riesgo apresurarme a las anteriores afirmaciones sin profundizar en lo que se entiende por grados de incertidumbre. Iniciemos con el supuesto de la validez de la propuesta, lo cual implicaría la existencia de una incertidumbre de mayor grado que se encontraría en la frontera borrosa entre el conocimiento y la ignorancia. Esta incertidumbre en "sumo grado" es frecuente encontrarla en la literatura sobre el riesgo. La cuasi-ignorancia o incertidumbre en sumo grado sobre los fenómenos estudiados es confundida con el riesgo mismo ${ }^{6}$. ¿Cómo hacer posible una efectiva divulgación del riesgo, si lo que divulgamos es nuestra ignorancia acerca de los fenómenos estudiados? ¿Era posible comprender el fenómeno del cambio climático, si existía más ignorancia que conocimiento acerca de sus causas y de sus efectos ${ }^{7}$ ?

6 Esta afirmación es fácil de corroborar en textos referidos a los llamados "riesgos globales". En su momento el cambio climático presentaba más incertidumbres cercanas a la ignorancia (en el contexto de la propuesta) que conocimientos acerca de sus causas o efectos, sin embargo era frecuente tratarlo como un riesgo más.

7 Hoy en día todavía existen dudas, pero el grado de conocimiento sobre los efectos del cambio climático es mayor. Existen estudios respaldados por una amplia comunidad de científicos de primer nivel que le dan carácter de riesgo a lo que antes era incertidumbre. 
En un grado menor de incertidumbre, se presume mayor conocimiento. En el contexto del riesgo los efectos se hacen evidentes, hay un tránsito de la incertidumbre al riesgo. Este tránsito se refleja en un cambio en las acciones a seguir, se cambia de la precaución a la prevención. En este estado de conocimiento (o de la incertidumbre) es posible divulgar ese grado de conocimiento, se hacen posibles y racionales las políticas públicas acerca del cuidado de nuestro medio ambiente. El riesgo es creado, representado y divulgado y la percepción del riesgo crece también en grado.

En la corriente fuerte, el riesgo, la incertidumbre y la ignorancia son grados de incertidumbre. Si quitáramos los apelativos, son incertidumbres. He ahí la confusión.

Renn (2005) propone una posible distinción entre incertidumbres que nos permite acercarnos a un primer debate y a aclarar la confusión generada en torno al concepto. Al respecto expresa,

Risk analysts consequently distinguish between aleatory and epistemic uncertainty: epistemic uncertainty can be reduced by more scientific research while aleatory uncertainty will remain fuzzy regardless of how much research is invested in the subject. Remaining uncertainties pose major problems in the later stages of risk characterization and evaluation as well as risk management since they are difficult to integrate in formal risk-benefit analyses or in setting standards.

(Renn, 2005, págs. 28-30)

Existen, entonces, dos clases de incertidumbre, una epistémica y otra de tipo estadístico. La primera de ellas podríamos asociarla a la corriente débil, pero reconociendo que con más investigación es posible reducirla tal como lo expresa el "principio de precau-

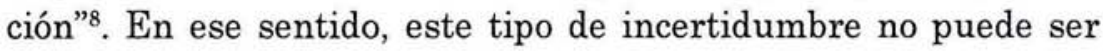

* La versión más popularizada es el principio 15 de la Declaración de Río sobre el Medio Ambiente y el Desarrollo (1992): "Con el fin de proteger el medio ambiente, los Estados deberán aplicar ampliamente el criterio de precaución conforme a sus capacidades. Cuando haya peligro de daño grave o irreversible, la falta de certeza científica absoluta, no deberá utilizarse como razón para postergar la adopción de medidas eficaces en función de los costos para impedir la degradación del medio ambiente". Falta de certeza absoluta es simple y llanamente: falta de conocimiento. 
igual al riesgo, es un concepto referido a la ignorancia o falta de conocimiento.

La incertidumbre estadística es un problema distinto, es el problema duro de resolver. Es la incertidumbre buscada o explicada desde los diferentes modelos estadísticos que permiten predecir posibles escenarios ${ }^{9}$. En dichos escenarios hay conocimiento de lo que podría suceder, pero no hay conocimiento de lo que con certeza sucederá. La incertidumbre se refiere al desconocimiento del futuro, no al desconocimiento de lo que ya es conocido. Es un concepto de incertidumbre más complejo que se vincula a otros igualmente complejos y debatidos como la probabilidad, la verdad, la certeza y la validez de un enunciado o proposición. En este tipo de incertidumbre es posible encontrar una luz en el túnel de la confusión.

2.1 Riesgo e incertidumbre. Dos conceptos entre la duda y la certeza

Furthermore, many potential impacts may be caused by a unique combination of events (surprises) which is impossible to predict. Thus uncertainty prevails in spite of the concerted effort to assess and manage risks.

(Zwick \& Renn, 2002, pág. 1)

La imposibilidad de predecir es una característica propia de otros fenómenos diferentes al riesgo (efectos de los transgénicos en el medio ambiente, por ejemplo). A estos fenómenos se responde con precaución. La posibilidad de predecir es una característica

9 La incertidumbre estadística, a su vez, presenta serias discusiones en torno a los observables y no observables. El debate Einstein-Bohr es un ejemplo de estas divergencias entre las incertidumbres de la estadística de la mecánica clásica (determinista, causal y predictiva en el lenguaje común, esto es, cuando se conoce exactamente el estado del sistema o se puede precisar exactamente el valor numérico de cada una de las magnitudes físicas como energía, momento cinético, posición, etc.) y las nuevas incertidumbres aleatorias de la mecánica cuántica (indeterminista y al azar). 
propia del riesgo. Obsérvese que no afirmo una predicción exacta. En situaciones de riesgo se predice los "posibles" futuros del mundo, dadas unas condiciones conocidas. Es posible que la predicción falle por la presencia de "incertidumbres", pero en últimas es predicción. Hay conocimiento de las causas y efectos que permiten estimar un futuro escenario, en el cual es posible recurrir a las probabilidades como base para la predicción. Al fallar la predicción surge el grado de incertidumbre que propone la corriente fuerte.

Si tratáramos de representar esta corriente y si aceptáramos las probabilidades como grados de creencia o de conocimiento, sería algo parecido a lo de la figura de la página siguiente ${ }^{10}$. El riesgo es un grado de incertidumbre, pero con probabilidades y efectos (costo-beneficio) conocidos. Podemos arriesgarnos a apostar un dinero a que el resultado del lanzamiento de una moneda es "cara", sabemos de su probabilidad (objetiva o subjetiva) y del costo-beneficio (perder o ganar el dinero) ${ }^{11}$; es decir, es posible predecir. En el otro extremo de la gráfica está la incertidumbre per se o si se prefiere, en grado sumo; en este caso es bajo o casi nulo el conocimiento de las probabilidades y de los resultados. En otros grados de incertidumbre existe conocimiento (así sea bajo) de alguno de estos dos factores. Sabemos que la probabilidad de una visita extraterrestre es baja o prácticamente nula (por experiencias no acaecidas), pero es incierto el costo-beneficio de esta visita. Desconocemos la probabilidad de un impacto de un gran objeto sobre la Tierra, pero sabemos de sus consecuencias ${ }^{12}$.

11 En el apartado 2.4 se profundiza en la probabilidad como grado de creencia.

"El común de la gente apuesta en el lanzamiento de una moneda con la creencia que su probabilidad de ganar es del $50 \%$.

12 Quizá en este último ejemplo se podría pensar en probabilidades bajas, posición relativa con respecto al tiempo. La Tierra ha sido impactada frecuentemente con grandes objetos a lo largo de su historia y por objetos pequeños en su historia más reciente. 


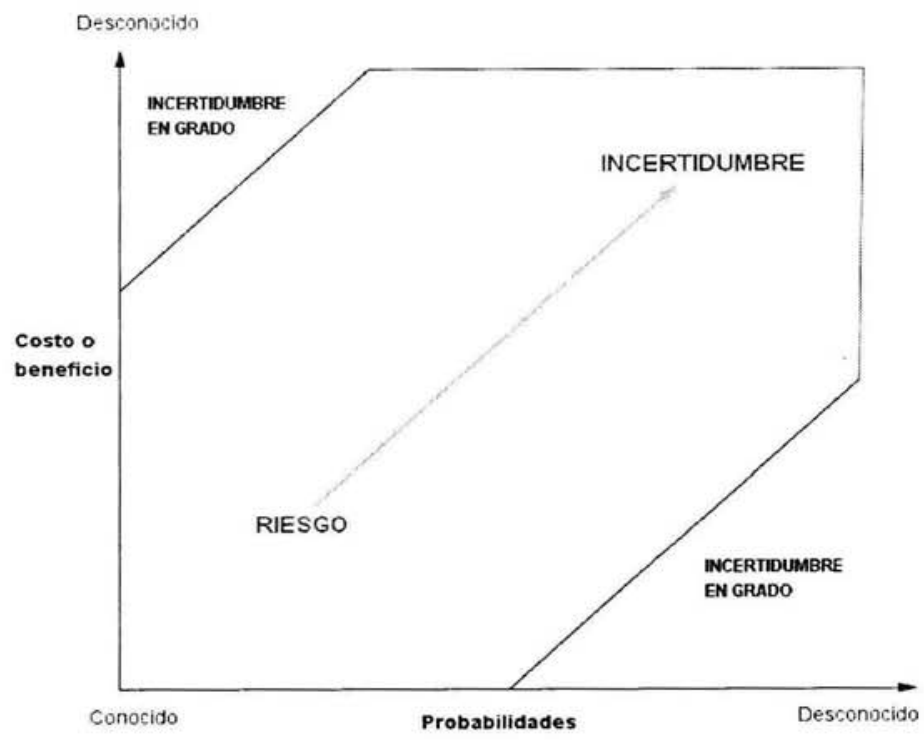

\subsection{Entre el conocimiento y la ignorancia. La borrosa distinción}

En un principio creí que mi hipótesis de una distinción riesgoincertidumbre era una lucha vana contra estas dos corrientes: la de una simbiosis de estos dos conceptos y la de la gradación de la incertidumbre. En ambas, el riesgo, si quitáramos sus adjetivos, es incertidumbre.

$\mathrm{Al}$ indagar en diversos referentes de la sociología, la psicología, la filosofía, la antropología y de tipo técnico o ingenieril, parecía ser que los bandos ya estaban definidos ${ }^{13}$. Sólo algunos casos aislados me alentaban a profundizar en lo que yo veía como evidente, así para otros resultara extraño.

13 El riesgo como incertidumbre es defendido, entre otros, por Rosa, Matsuda y Kleinhesselink (Renn \& Rohrmann, 2000, pág. 188). Rosa (1998, pág. 28) afirma: Risk is a situation or event uhere something of human value (including human themselves) has been put at stake and where the outcome is uncertain. Giddens denomina el riesgo como una "incertidumbre fabricada" (Beck, Giddens, \& Lash, 1997 (1994), pág. 220). 
Un primer acercamiento a esta distinción se elucida en la corriente fuerte. López-Cerezo y Luján (2000) establecen una tipología para la incertidumbre: riesgo, incertidumbre, ignorancia e indeterminación. En la primera categoría, la incertidumbre denominada riesgo requiere del conocimiento de la probabilidad de uno u otro resultado; mientras que para la segunda categoría, la incertidumbre per se, no se conoce una posible distribución de probabilidades. La incertidumbre es, entonces, para estos dos autores, la carencia de probabilidades. A pesar de la taxonomía, hay distinción. Los autores dejan entrever un conocimiento, así no sea muy concreto, al proponer posibles efectos, bajo incertidumbre. Es decir, así se denomine incertidumbre en grado, hay conocimiento. Mi propuesta es la de un concepto de riesgo en el conocimiento, el cual me permite predecir posibles efectos y tomar decisiones racionales en torno a dicho conocimiento. Voy a defender esta hipótesis en la cual el riesgo se construye desde el conocimiento en contraposición a la incertidumbre que se recrea en las categorías de la ignorancia, donde no es posible predecir. En la ignorancia no existe nada que podamos clasificar, definir o ponernos de acuerdo. Mientras no haya distinción, no habrá acuerdo.

$\mathrm{Si}$, bien, López-Cerezo y Luján retoman esta tipología de Wynne (1992), dejan a un lado tres categorías que Wynne incluye: complejidad, desacuerdo y ambigüedad. Categorías que, más que justificar la gradación propuesta, justifican una distinción riesgo-incertidumbre. La complejidad entendida como un grado de incertidumbre es frecuente cuando la primera se reduce mediante modelos inadecuados o incompletos de cadenas causa-efecto. $\mathrm{Al}$ respecto Renn (2005, pág. 30) plantea,

Uncertainty is different from complexity but often results from an incomplete or inadequate reduction of complexity in modeling cause-effect chains. Whether the world is inherently uncertain is a philosophical question that we will not pursue here.

En el desacuerdo no hay un concepto de incertidumbre sobre el que podamos discutir, es el problema que estamos afrontando, así que queda subordinado al resto de este apartado. 
En la ambigüedad se pierde el sentido de ambos conceptos, es aquí donde se hace borrosa la distinción. Modelar la incertidumbre con los paradigmas de la "Utilidad Esperada", con estudios sofisticados desde la estadística estocástica o desde la novedosa lógica borrosa o difusa, sólo ha aumentado la confusión entre incertidumbre y riesgo ${ }^{14}$. Si aceptáramos como válida la premisa de que el riesgo se evalúa desde las probabilidades, podemos asegurar que no hablamos de una lógica bivalente. $\mathrm{Al}$ igual que la lógica difusa, el estudio de las probabilidades no se circunscribe a dos valores de verdad. Una probabilidad de un $80 \%$ de contraer un virus, no dice que es "verdadera" la infestación por este virus. Una probabilidad de un $80 \%$ de contraer un virus dice que "es casi cierta" dicha contaminación. ¿En qué se diferencia de la lógica difusa? ¿Dónde surge la confusión? Si asumiéramos la palabra "posibilidad" en lugar de "probabilidad", es decir, la probabilidad en términos de "grados de creencia", no tendríamos diferencia con los conjuntos difusos, los cuales son una distribución de posibilidades en términos de los llamados "grados de pertenencia"15.

Sin necesidad de profundizar en los extensos tratados sobre lógica difusa y de los aún más extensos tratados de lógica no bivalente anteriores a la primera, no es dificil identificar los principios que orientaron la propuesta de esta "novedosa" lógica. Cohen y Hansel (1956) hacen alusión al lenguaje de la incertidumbre en palabras o frases como: "períodos de bajo rendimiento", eventualmente,

14 Los debates en torno a las probabilidades se abordarán en las siguientes secciones de este apartado. La separación es intencional, dado que me apoyaré en la discusión actual para fundamentar mi hipótesis de una distinción necesaria entre riesgo e incertidumbre de cara a un consenso conceptual.

15 En principio, puedo reñir fuertemente con los estudiosos de la lógica difusa, ofrezco excusas y a la vez pido licencia para continuar con mi argumentación, pues el concepto de probabilidad también entrará en discusión y, finalmente, podremos estar hablando de lo mismo. Es decir, es "posible" que estemos de acuerdo. Léase Zadeh (1965) para el concepto original de grados de pertenencia (grades of membership). En Matos (2007) se presenta una propuesta multi-criterio de análisis de la incertidumbre, la cual incluye modelos de distribución probabilística y de distribución "posibilística" o difusa. Véase también Dubois (2006). 
ordinariamente, "alto desempleo", "algunas enfermeras", "muy bajo", etcétera. Poco después, Zadeh (1965) afirmó que clases como "las mujeres bellas" o "los hombres altos" no pueden tratarse dentro de la teoría clásica de conjuntos, sino como clases en grados de pertenencia (más bella, menos bella, más alto,...) ${ }^{16}$. La "distribución de posibilidades" de cada uno de esos elementos "difusos" es la colección de los posibles valores de dichos elementos (Zadeh, 1981). Esta lógica borrosa o difusa es una respuesta a la vaguedad o, para ser más precisos, a la imprecisión. Es incertidumbre de la medida, lo cual implica conocimiento de lo impreciso.

Probabilidad o posibilidad es quizá otra fuente de confusión. Zadeh (1984, pág. 363) mezcló estos dos conceptos en afirmaciones como,

In the conventional approaches to decision analysis, uncertain information is treated probabilistically, with probabilities assumed to be known in numerical form. In an alternative approach [...], a more realistic assumption is made, namely, that probabilities are known imprecisely as fuzzy rather than crisp numbers. Such probabilities - which will be referred to as fuzzy probabilities- are exemplified by the perceptions of likelihood which are commonly labeled as very likely, unlikely, not very likely, etc.

Se confunde probabilidad con posibilidad.

En la propuesta de gradación de la incertidumbre presentada por Funtowicz y Ravetz (2001), en la Ciencia Posnormal, se destaca la incertidumbre en grado sumo. En sus argumentos, Funtowicz y Ravetz apelan al problema que hoy en día enfrentan científicos de todas las disciplinas, el problema de las incertidumbres globales, especialmente las de tipo ambiental enunciadas por Beck ${ }^{17}$. En este

16 Véase Zadeh (2005, pág. 166) sobre la imprecisión basada en el conocimiento y Zadeh (2006, págs. 15-16) sobre la teoría generalizada de la incertidumbre. En esta última, Zadeh inicia con esta frase: Uncertainty is an attribute of information.

${ }_{17}$ Beck inició su discusión sobre los "riesgos globales" con la publicación del libro Risk society: Towards a new modernity. Destaca la aparición de riesgos invisibles, riesgos no naturales, riesgos incontrolables, que tienen su origen en la creación, distribución y uso de las nuevas tecnologías. 
tipo de incertidumbre se devela la debilidad de la empresa científica, su incapacidad de predicción y, por consiguiente, su distinción con el riesgo. A pesar de la propuesta de gradación, al igual que Beck, reducen las incertidumbres en grado sumo a riesgos globales. Esta reducción, que no comparto, permite que la confusión entre riesgo e incertidumbre siga latente. Al igual que López-Cerezo y Luján, la incertidumbre no entra en el ámbito de la ignorancia, sino en las cercanías de la ignorancia, al respecto expresan,

Thus, these experts discover in their own practice an extreme form of uncertainty, on that borders on ignorance [...] uncertainty cannot be treated by standard mathematical or computational techniques.

Funtowicz y Ravetz (2001, págs. 188-189)

Hubiese sido más simple traspasar la frontera y llevar la incertidumbre al mundo de la ignorancia, no habría confusión. Rescato la posición de una incertidumbre que no se puede medir a través de modelos matemáticos comunes.

Degradar la incertidumbre en términos del conocimiento es cambiar el sentido mismo de la incertidumbre. Una gradación de la incertidumbre resulta ser inconveniente para el propósito de alcanzar un acuerdo conceptual en torno al riesgo.

La incertidumbre es duda, el riesgo es posibilidad. Responder "no sé" a la pregunta: ¿es posible que haya pérdidas humanas?, es una respuesta desde la incertidumbre, una respuesta positiva se da desde del conocimiento y cualquier decisión es bajo riesgo. Renn (2005, pág. 30) acepta una falta de consenso en cómo se puede lograr esta gradación. Sin embargo, propone cinco categorías de incertidumbre, dos de las cuales coinciden con las propuestas por Wynne: Indeterminación e Ignorancia ${ }^{18}$. Es claro que la ignorancia es la categoría en la cual estará el consenso.

18 Renn propone cinco categorías, entre las cuales se destaca (a pesar de esquivar una discusión filosófica) el riesgo inductivo enunciado por Carnap. Riesgo que describe como error en la modelación. Para ello, cita como ejemplo la modelación para seres humanos partiendo de aplicaciones en animales. Este riesgo inductivo lo clasifica, además, como una "incertidumbre epistémica". 


\title{
2.3 La probabilidad como factor de desacuerdo
}

\begin{abstract}
All our philosophy is a correction of the common usage of words ${ }^{19}$.
\end{abstract}

(von Mises, 1981, pág. 1)

La frase con la que von Mises (1981) inicia su defensa del concepto de probabilidad como palabra clave que permite la relación entre la estadística y la verdad científica se constituye, en mi concepto, en una expresión que refuerza la importancia de las palabras y su significado en el lenguaje científico. Riesgo, incertidumbre, probabilidad y creencia, amplían la compleja red de conceptos en los cuales tendremos que profundizar para comprender la falta de consenso en uno u otro concepto. Cada uno de ellos se utiliza con connotaciones distintas en un contexto específico o en un paradigma determinado y, sin embargo, son utilizados en un lenguaje común para representar, informar o comunicar las características de un fenómeno cualquiera. "Es probable que mañana llueva", es una frase que para un grupo de personas tengan significados distintos. Para un experto climático, dentro de su paradigma, existen suficientes razones para creer que el comportamiento del clima en el día de mañana sea lluvioso. Para un matemático no dice nada, en tanto no se conozca el modelo probabilístico utilizado y las fuentes clave que permitan un estimador más robusto. Para un filósofo escéptico, la palabra probabilidad no existe o se trata de una simple especulación. Para un hombre común existen suficientes razones, dadas por la experiencia, para dudar de esta afirmación ${ }^{20}$.

19 Esta frase es tomada de uno de los aforismos del científico alemán Georg Christoph Lichtenberg. A pesar de ser una expresión del siglo XVIII, von Mises la utiliza como introducción a su extensa defensa del concepto de probabilidad frecuentista y, agrega: "Muchas de las discusiones y de los errores que ocurren en el curso del avance científico podrían ser evitados si esta observación fuera recordada siempre".

20 La ya conocida desconfianza en los científicos se incrementa con los errores predictivos del clima. 
El problema de múltiples significados de una sola palabra trae como consecuencia la incomprensión o distorsión del mensaje que se pretende hacer llegar. Esta situación se hace más compleja cuando dentro de la comunidad científica no hay acuerdo en la definición de la palabra. Para hacer más complejo el problema, en el contexto de los países de habla hispana, solemos incorporar del idioma inglés palabras como: Chance, likelihood, probability, odds, gamble, feasibility, likeliness y prospect con significados similares y, a veces, sinónimos ${ }^{21}$. Agravando aún más la situación, se tiende a confundir con el mismo concepto de incertidumbre: "La verdad probable o contingente abarca todos aquellos juicios que dependen de sucesos cuyas características no podemos investigar y cuya predicción se hace con un alto grado de duda" (Devey, 1854, pág. 166) ${ }^{22}$.

Pero el panorama no es tan oscuro. Si Devey asociaba la probabilidad a la incertidumbre, contemporáneos como De Morgan (1847, págs. 170-173) y Boole (1854, pág. 244) la asociaban a grados de creencia ${ }^{23}$. Postura que es defendida por Keynes (1921, pág. 20) como grados de creencia racional y por los llamados bayesianos (Ramsey, Savage y de Finetti).

\subsection{La probabilidad como factor de distinción}

Philosophical efforts to clarify this concept are as old as its history, and the basics ideas of the two main groups of theories which seek to determine its meaning go back to the beginnings of this history. For a first orientation, these may be contrasted as the "aprioristic" an "empiricist" views of probability.

Hempel (1965, pág. 89)

21 Rivadulla (1991, pág. 261) describe esta tendencia de asignarle diferentes palabras al concepto de probabilidad: "[...] también Leibniz, quien en De incerti aestimatione', 1678 , concibe la probabilidad como 'gradus possibilitatis' utiliza con frecuencia la expresión' estimación' de probabilidad; y John Arbuthnot, quien en 1692 publica la traducción del libro de Huygens, con el título de 'Of the laws of Chance', traduce como 'equal probabilities' la expresión 'posibilidades iguales'.

${ }_{22}$ Un alto grado de duda no es más que incertidumbre en grado sumo.

23 De Morgan afirmaba que grados de conocimiento y grados de probabilidad son grados de creencia, donde la creencia es conocimiento imperfecto. Jakob Bernoulli, en 1713, empleaba la expresión "grados de certeza". 
Asumiendo como válidas las categorías de Wynne y entendiendo que la mayor confusión se da en la incertidumbre aleatoria, el referente obligado para establecer la distinción riesgo - incertidumbre es entonces el concepto de probabilidad. Es allí donde encuentro un primer acuerdo con algunas autoridades. Knight (1921) fue uno de los primeros investigadores del riesgo en proponer una distinción entre riesgo e incertidumbre ${ }^{24}$. Para ello, habla de dos tipos de incertidumbre, una mensurable y otra inmensurable. La primera, la asocia al término "objetivo" y al concepto de riesgo. Por otra parte, las probabilidades subjetivas y la incertidumbre inmensurable es lo que entiende Knight por incertidumbre propiamente. Esta postura, no gradada, sobre la incertidumbre es respaldada, en parte, por Elster (1990). Plantea Elster que en la incertidumbre, el agente o conjunto de agentes, no puede asignar probabilidades numéricas a la ocurrencia de un suceso, tampoco puede especificar el conjunto completo de posibles estados del mundo, y ni siquiera es capaz de dar cuenta de las probabilidades de estos estados en el futuro. Tomar decisiones bajo incertidumbre, es tomar decisiones desde la ignorancia. En este punto hay dos acuerdos de mi hipótesis con las posturas de Knight y Elster. El primero, referido a la necesidad de una distinción y el segundo, en un concepto de riesgo construido desde el conocimiento de las probabilidades ${ }^{25}$. Estoy más de acuerdo con Elster en una incertidumbre como reflejo de la ignorancia. Si bien Knight defiende la distinción riesgo-incertidumbre, su concepto de incertidumbre inmensurable es subordinado a las probabilidades subjetivas, en las cuales, sin embargo, hay conocimiento subjetivo ${ }^{26}$.

24 Frank H. Knight fue uno de los fundadores de la escuela de Economía de Chicago. Según Knight, el riesgo está referido a una situación donde la probabilidad de un resultado está determinado. En contraste, en la incertidumbre se presentan eventos cuyas probabilidades son desconocidas.

25 En lugar de probabilidades, estoy más de acuerdo con la palabra "posibilidades" (likelihood en inglés), afirmación que sustentaré en mi propuesta de noción de riesgo.

${ }^{26}$ Las probabilidades objetivas o subjetivas tienen un componente subjetivo. Afirmación que defiendo en el apartado siguiente. 
El concepto de probabilidad asociado al concepto de riesgo presenta problemas de consenso entre los diversos tipos de probabilidad. Nuevamente, se justifica la profundización en las raíces del desacuerdo. Así como actualmente enfrentamos el problema de una multiplicidad de conceptos de riesgo y de incertidumbre, el concepto de la probabilidad no escapa a las múltiples concepciones. Carnap (1969 (1966), pág. 39) en este sentido se lamentaba,

... la mayoría de los libros sobre la probabilidad no establecen una distinción entre diversos tipos de probabilidad, una de las cuales sea llamada la "probabilidad lógica". [...] Es lamentable que se haya usado la misma palabra, "probabilidad", en dos sentidos tan diferentes. Por no realizar esta distinción surgen enormes confusiones en libros sobre filosofia de la ciencia y en declaraciones de los mismos científicos.

Obsérvese que en la expresión de Carnap y en el contexto de las categorías de Wynne, podríamos parodiar: ... por no realizar la distinción riesgo-incertidumbre surgen enormes confusiones... Establecer esta distinción coadyuva a un acercamiento al concepto de riesgo. Carnap planteaba la existencia de dos tipos de probabilidad, una estadística y otra lógica. Es decir, por inferencia estadística o por inferencia inductiva (no demostrativa) ${ }^{27}$. Los tipos de probabilidades a las que hace alusión Carnap, a su vez, permiten profundizar en la complejidad de la incertidumbre.

La probabilidad lógica de Carnap surge como un método para definir lo que denominó "grado de confirmación" para cualquier hipótesis, expresada con relación a una información dada (Hempel, 1979 (1966), pág. 75). Pese a esta clasificación de Carnap, es frecuente encontrar otras taxonomías sobre la probabilidad. Al debate entre keynesianos y frecuentistas se suman otros debates igual de importantes por su contenido epistémico: Bayesianismo versus frecuentismo, probabilistas (objetivistas) versus subjetivistas y,

${ }_{27}^{27}$ Asumiré como inferencia inductiva aquella que implica una conclusión con un grado más o menos alto de probabilidad, mientras que las conclusiones por inferencia deductiva son conclusiones con certeza (Hempel, 1979 (1966), pág. 27). 
en forma más particular, creencia versus preferencia. Sea cual fuere el concepto de probabilidad, tanto en su construcción como en el modelo adoptado, la creencia juega un papel importante ${ }^{28}$. La probabilidad per se es subjetiva.

Ramsey, al igual que Carnap, abogaba por una distinción entre las probabilidades lógicas y estadísticas. Según Ramsey, una y otra escuela discuten en torno a conceptos distintos,

Indeed the general difference of opinion between statisticians who for the most part adopt the frequency theory of probability and logicians who mostly reject it renders it likely that the two schools are really discussing different things, and that the word 'probability' is used by logicians in one sense and by statisticians in another. The conclusions we shall come to as to the meaning of probability in logic must not, therefore, be taken as prejudging its meaning in physics.

Ramsey 1925, p. 157

¿Por qué surgen estos debates? La respuesta a este interrogante refuerza aún más la importancia de una distinción riesgo-incertidumbre. Los debates tienen su trasfondo en la formulación de teorías que tratan de dar respuesta a los fenómenos del mundo. La validez de las teorías se fundamenta en la validez de las leyes que la constituyen. Las leyes a su vez, en palabras de Carnap, tienen grados de confirmación que se traducen en grados de creencia ${ }^{29}$.

28 Se excluyen las probabilidades objetivas de Popper o las que denominó "posibilidades con peso" o propensiones (Popper, 1996 (1990)). Las propensiones que defiende Popper son realidades físicas, no son inherentes al sujeto, están más allá de nuestras creencias. La propensión tampoco es inherente al objeto, según Popper es inherente a la situación, donde el objeto es parte de la misma. Pero, sean físicas o no, las propensiones de Popper también son una cuestión de grado, más de posibilidad que de probabilidad: "Ni nuestro mundo ni nuestras teorías físicas son determinísticas, aun cuando no quepa duda de que las leyes de la naturaleza y de la probabilidad excluyen muchas posibilidades: hay un buen número de posibilidades cero. Es más, las propensiones distintas de cero pero de valor muy pequeño no se realizarán si la situación cambia antes de que tengan la ocasión". (Popper, 1996 (1990), pág. 51).

29 Me refiero a las leyes empíricas tal como lo concibe Carnap (1969 (1966), págs. 2425), exceptúo las leyes de la lógica y de la matemática pura, o las llamadas leyes universales, excepción que se justifica en el carácter de certeza de estas leyes. Las 
En estos grados de creencia entran las probabilidades a ejecutar su rol. Para no alargar la discusión en el campo filosófico, partiré de una simple afirmación: "la probabilidad es un grado de creencia"30. Grado de creencia como contraposición al "grado de incertidumbre". Medir probabilidades es medir creencias,

The subject of our inquiry is the logic of partial belief, and I do not think we can carry it far unless we have at least an approximate notion of what partial belief is, and how, if at all, it can be measured. It will not be very enlightening to be told that in such circumstances it would be rational to believe a proposition to the extent of $2 / 3$, unless we know what sort of a belief in it that means. We must therefore try to develop a purely psychological method of measuring belief. It is not enough to measure probability; in order to apportion correctly our belief to the probability we must also be able to measure our belief.

Ramsey 1925, p.166

La probabilidad como grado de creencia se devela tanto en las llamadas probabilidades objetivas y, como es obvio, en las subjetivas. La forma más simple de probabilidad objetiva y más temprana en la historia de las probabilidades es el caso que se da en un espacio muestral finito y con resultados equiprobables. Desde el concepto clásico de la probabilidad, aún vigente, es común representarla con expresiones, tales como: $\mathrm{P}(\mathrm{A})=\mathrm{Rf} / \mathrm{Rp}$, donde $\mathrm{Rf}$ son los resultados favorables del evento $\mathrm{A}$ y $\mathrm{Rp}$ los resultados posibles (Carnap, 1969 (1966), pág. 40). Por ejemplo, la probabilidad que el resultado de un lanzamiento de una moneda sea cara es $\mathrm{P}(\mathrm{A})=1 / 2$ o del $50 \%$. Esta afirmación está cargada de creencia. Creencia en la

leyes empíricas o probabilísticas (Hempel, 1979 (1966), pág. 93) son comunes en la formulación de teorías y principios de las ciencias naturales.

30. La discusión en este sentido es mucho más amplia. Un ejemplo es el debate PopperCarnap entre verificación y corroboración, así Carnap posteriormente acuda al concepto de confirmación. No pretendo ser reduccionista con mi afirmación, pretendo evitar una profundización en conceptos que no ayudan o no agregan más a mi base argumentativa. Tampoco es una declaración de afinidad al bayesianismo; mi afirmación es general, para cualquier tipo de probabilidad o teoría probabilista. 
equiprobabilidad, creencia en las características homogéneas de la moneda y, especialmente en la inferencia estadística, generadora a su vez de innumerables debates ${ }^{31}$.

La inferencia estadística así como la inferencia inductiva son fruto de la creencia, no de la incertidumbre. En lo incierto, como lo expresa Elster, no hay creencia. No es posible creer en lo que se ignora ${ }^{32}$. Las leyes bajo certeza son universales, las leyes empíricas nacen de la creencia como grado. El grado de creencia de obtener cara en el lanzamiento de una moneda es del $50 \%$, el riesgo es del $50 \%$. El grado de creencia de resultar afectado por una epidemia gripal es alto, la creencia construye el riesgo. Reducir la incertidumbre o la ignorancia es convertirla en "razones para creer", es llevarla al nivel del riesgo. No hay incertidumbre de morir en una explosión atómica, hay riesgo y, por contraste, es demasiado alto, tan alto que se convierte en certeza. Existe un alto grado de creencia que hemos denominado certeza o, ley universal. Desde la incertidumbre gradada o no, no es posible formular una ley, menos una teoría que dé cuenta de lo que no conozco. No es posible formular una ley empírica que dé cuenta del comportamiento humano en el caso de una invasión extraterrestre, ni causa ni efecto tienen referente empírico.

Apostarle a la incertidumbre como riesgo es confundir el concepto mismo de riesgo. Apostarle a la incertidumbre como grado igualmente confunde. Ravetz (1997, pág. 6) se refiere a la incertidumbre como,

Formerly, theorists distinguished between "risk", which could be quantified, and "uncertainty", which could not. Now we have various kinds and degrees of uncertainty, and ignorance as well. And even ignorance reveals a host of varieties within itself. In particular, we have "policy-critical ignorance", as when

31 Un primer cuestionamiento a esta probabilidad simple la plantea Bernoulli mediante la conocida paradoja de San Petersburgo enunciada en 1738. He ahí una probabilidad objetiva cargada de subjetividad.

32 Excluyo las creencias fruto de la fe, las cuales se anclan en un conocimiento de tipo cultural. 
there are no parameters, no theories, hardly any guesses, to supply a necessary foundation for policy decisions.

Igualmente confunde.

Las teorías se introducen normalmente cuando estudios anteriormente realizados de una clase de fenómenos han revelado un sistema de regularidades que se pueden expresar en forma de leyes empíricas. Las teorías intentan, por tanto, explicar estas regularidades y, generalmente, proporcionar una comprensión más profunda y exacta de los fenómenos en cuestión.

\section{UN ACERCAMIENTO A LA DISTINCIÓN RIESGO-INCERTIDUMBRE DESDE LAS CREENCIAS}

Thus, both doubt and belief have positive effects upon us, though very different ones. Belief does not make us act at once, but puts us into such a condition that we shall behave in some certain way, when the occasion arises. Doubt has not the least effect of this sort, but stimulates us to action until it is destroyed

(Peirce, 1877)

Mucho se ha escrito sobre las creencias. Por contraste a los conceptos de riesgo, de incertidumbre y de probabilidad, el concepto de "creencia" es más nítido, menos borroso. A pesar de su gradación.

La acción de creer es el fin de la duda, es la eliminación de la incertidumbre. Con la creencia cesa la lucha que causan nuestras dudas o incertidumbres (Peirce, 1877). Creer en las teorías, creer en los postulados de una corriente de pensamiento, creer en los dogmas de una religión, creer en nuestras opiniones, es crear nuestra red de conceptos y por consecuencia nuestro conocimiento subjetivo. Hay certeza en nuestras creencias, todas ellas, para nosotros, son verdaderas... así sean falsas. Es verdad que Dios existe; tengo certeza en que si el terremoto es de grado 8.0, mi casa sufrirá graves consecuencias; creo que Bush es un excelente gobernante; no creo en incertidumbres gradadas o dudas pequeñas o grandes, todas ellas son "no conocimiento" y como tal, no las puedo medir... esa es mi 
creencia. Pero este conocimiento es por grado, se hace de mayor o de menor grado en tanto interactuemos, para compartir nuestras creencias. Esta gradación va de muy ignorantes a muy sabios, de legos a expertos. La interacción con grupos sociales, culturales o científicos reafirman o cambian nuestras creencias. Es en esta interacción en la que creamos el conocimiento objetivo. La creencia dominante es la creencia intersubjetiva. Pero esta dominación se vuelve insoportable para algunas de nuestras creencias. Surgen de nuevo las dudas, se presentan otras luchas internas por lograr la creencia. El objeto de estas luchas no es la incertidumbre, es algo que pertenece a nuestra red de conceptos o a nuestra esfera de conocimientos: "And it is clear that nothing out of the sphere of our knowledge can be our object, for nothing which does not affect the mind can be the motive for mental effort" (Peirce, 1877).

La reflexión anterior la hago para ubicar el concepto de creencia como concepto de conocimiento. Verdadero o falso, pero en últimas nuestro conocimiento. Creer en la existencia de incertidumbres es creer que existe la ignorancia, esto es verdadero, es tautológico. Pero creer que puedo medir el "no conocimiento", es una contradicción. ¿Cuánto no sé de lo que no sé? es una pregunta sin sentido. Lo que realmente medimos es nuestra creencia con respecto a la creencia dominante. Medimos nuestro conocimiento con referencia al conocimiento paradigmático, al conocimiento social, cultural, político o científicamente aceptado como creencia válida. Podemos medir nuestro grado de conocimiento (grado de creencia) en mecánica cuántica, en la geografía de Europa o sobre el cambio climático. No podemos medir nuestro "no conocimiento" sobre los impactos del cambio climático.

Cuando Heisenberg enuncia su "principio de incertidumbre", lo hace no como "principio de no conocimiento", sino en términos de "imposibilidad de determinar" dos variables físicas inherentes a una partícula simultáneamente ${ }^{33}$. Pero, hay toda una esfera

33 Este principio se constituye en uno de los baluartes de la teoría cuántica. El principio formulado por el alemán Werner Heisenberg demuestra que a nivel cuántico no es posible conocer de forma exacta el momento lineal y la posición de una partícula: 
de conocimientos en torno a la partícula, conocimiento sobre la velocidad y la posición. Podemos medirlas, sin embargo, no podemos hacerlo simultáneamente ${ }^{34}$. ¿Existen dudas sobre la existencia de la partícula, de su posición y su velocidad? Lo que existe es una imposibilidad en su medición y es por ello que se prefiere enunciar el principio como "principio de indeterminación". La indeterminación referida al proceso de medición y por ende al proceso de predicción. $\mathrm{Ni}$ en la incertidumbre, ni en la indeterminación podemos predecir. Desde el conocimiento o desde nuestras creencias podemos predecir, obteniendo como resultados nuevas creencias. En ese contexto se crea el riesgo. El riesgo es creencia en que es posible que algo suceda $^{35}$. La creencia en que algo sucederá, no es riesgo, es certeza. En la frase "yo creo que si una bala me da en la cabeza, la bala me herirá", hay certeza. En la frase "yo creo que si el terremoto acaece con una magnitud de 8.0, mi casa posiblemente colapse" hay un grado de creencia, se crea el riesgo. En contraposición a esta última creencia, existe otra de menor grado, que la casa no colapse.

¿Cómo medir la creencia? La respuesta a esta pregunta, al parecer, se encuentra en la probabilidad bayesiana. No obstante, como lo he afirmado antes, las probabilidades objetivas no escapan a la subjetividad propia de los diferentes modelos aplicados. No es extraño que en torno a un modelo "objetivo" esté presente el debate, el cual es consecuencia de los grados de creencia de uno u otro actor del debate.

\footnotetext{
"At the instant of time when the position is determined, that is, at the instant when the photon is scattered by the electron, the electron undergoes a discontinuous change in momentum. This change is the greater the smaller the wavelength of the light employed, i.e., the more exact the determination of the position. At the instant at which the position of the electron is known, its momentum therefore can be known only up to magnitudes which correspond to that discontinuous change; thus, the more precisely the position is determined, the less precisely the momentum is known, and conversely (Heisenberg, 1927, p. 174-5).

3. La incertidumbre asociada a las probabilidades es criticada por Popper al enunciar sus discrepancias con Heisenberg, Einstein y otros físicos que concebían las probabilidades como algo que tenía que ver con la ausencia de conocimiento (Popper, 1996 (1990), pág. 23) .

35 Nótese que hablo de posibilidad, no de probabilidad.
} 
En este contexto es importante revisar lo que se entiende por probabilidad bayesiana, a pesar de las objeciones planteadas por los objetivistas o frecuentistas,

There is a continuing debate in the philosophy of probability between subjectivist and objectivist views of uncertainty. Objectivists identify probabilities with limit frequencies and consider subjective belief as scientifically irrelevant. Conversely subjectivists consider that probability is tailored to the measurement of belief, and that subjective knowledge should be used in statistical inference. Both schools anyway agree on the fact that the only reasonable mathematical tool for uncertainty modelling is the probability measure.

Dubois (2006, pág. 47)

Una de las principales discusiones de los bayesianistas es que la incertidumbre se debe representar por la probabilidad (Dubois, Prade, \& Smets, 1996). Afirmaciones como la anterior son frecuentes en muchos de los textos que hacen alusión a las probabilidades bayesianas. Pero, ¿el objetivo de los bayesianistas es medir la incertidumbre? Para los tempraneros exponentes como de Finetti, Ramsey y Savage, la probabilidad de un suceso mide es el grado de creencia en la ocurrencia de dicho suceso, percibido por un sujeto ${ }^{36}$. Ramsey (1931) reconoce en principio que la medición de la creencia no es fácil y que ésta puede ser medida hasta cierto punto; plantea que algunas son más medibles que otras, depende de la medida. Tal sistema es satisfactorio si logra asignar a cualquier creencia una magnitud o grado, lo cual significa introducir cierta cantidad de hipótesis o de ficción, tal como ocurre en otros sistemas,

36 Taroni et al (2006) definen el grado de creencia como el grado personal de creencia en que una proposición que describe un hecho, sea verdad. Estos autores prefieren hablar de grados de creencia débiles o fuertes. Posición con la que estoy de acuerdo. A una teoría subjetiva, no podemos cargarla con más subjetividad, pretendiendo establecer un sistema de medición que difícilmente pueda diferenciar una creencia de grado $1 / 16$ con otra de grado $1 / 8$. 
Even in physics we cannot maintain that things that are equal to the same thing are equal to one another unless we take 'equal' not as meaning 'sensibly equal' but a fictitious or hypothetical relation

Ramsey (1931, pág. 168)

En la propuesta de Ramsey se asigna 1 a una creencia total (certeza), 0 a su contradicción o complemento (incertidumbre en grado sumo) y para creencias iguales en una proposición y su contradicción (incertidumbre en grado), se asigna 1/2. Para otros grados la tarea se complica, pero insiste en que es absolutamente necesaria,

But it is not so easy to say what is meant by a belief $2 / 3$ of certainty, or and it's contradictory by a belief in the proposition being twice as strong as that in its contradictory. This is the harder part of the task, but it is absolutely necessary; for we do calculate numerical probabilities, and if they are to correspond to degrees of belief we must discover some definite way of attaching numbers to 1 degrees of belief. In physics we often attach numbers by discovering a physical process of addition.

Ramsey (1931, pág. 169)

El término probable bayesiano significa, entonces, el grado de creencia en un hecho como cierto ${ }^{37}$. Si no se sabe si ocurrió el hecho, sólo un grado de creencia menor que la certeza se puede asignar a la ocurrencia del hecho, tal como lo describe Peirce,

37 Venn (1876, págs. 104-107), frecuentista por convicción, no estuvo de acuerdo con una ciencia de la probabilidad vinculada a las leyes de la creencia. Acepta la extraordinaria analogía entre creencia o no creencia en un suceso con las probabilidades 1 ó 0 de que suceda. Lo que no estuvo dispuesto a admitir es la analogía de las probabilidades con creencias parciales. Critica y no acepta las posturas contrarias como la de De Morgan (1847, págs. 170-191), éste afirmaba que: "By degree of probability we really mean, or ought to mean, degree of belief". Lo que resalto y comparto con De Morgan es la afirmación de unos grados de probabilidad como grados de creencia. 
The numbers one and zero are appropriated, in this calculus, to marking these extremes of knowledge; while fractions having values intermediate between them indicate, as we may vaguely say, the degrees in which the evidence leans toward one or the other.

(Peirce, 1923, pág. 64)

Uno de los conceptos en los que se fundamentan los bayesianistas es el referido a la probabilidad condicionada,

Instead of speaking of this as the probability of the inference, there is not the slightest objection to calling it the probability that, if A happens, B happens. But to speak of the probability of the event $B$, without naming the condition, really has no meaning at all.

(Peirce, 1923, pág. 69)

Esta relación causa-efecto se suele representar por expresiones como $\mathrm{p}(\mathrm{B} / \mathrm{A})$. Bayes generaliza la expresión representando la cadena causal para un evento $B$ de la siguiente manera:

Si $A_{1}, A_{2}, \ldots \ldots ., A_{n}$ son un conjunto de eventos que forman una partición del conjunto de posibilidades de un fenómeno cualquiera, tal que $0<p\left(A_{i}\right)<=0, \forall i=l \ldots n$, entonces, para un evento $\mathrm{B}$ cualquiera en el espacio muestral de posibilidades de ocurrencia se verifica:

$$
p\left(A_{i} \mid B\right)=\frac{p\left(A_{i}\right) p\left(B \mid A_{i}\right)}{p\left(A_{1}\right) p\left(B \mid A_{1}\right)+p\left(A_{2}\right) p\left(B \mid A_{2}\right)+\ldots+p\left(A_{n}\right) p\left(B \mid A_{n}\right)}
$$

y esto para cualquier $i=1, \ldots ., n$.

Esta generalización o "teorema de Bayes" muestra una dependencia del evento $B$ con las causas $A_{i}$, cuyas probabilidades son conocidas $^{38}$. Es decir, el grado de creencia de que el evento B

38 Existen modelaciones mucho más complejas que mi representación de la probabilidad bayesiana. Por ejemplo, De Finneti y Ramsey se refieren a eventos lógicamente relacionados entre sí, los cuales se deben regir por las reglas del cálculo de probabilidades y formulan modelos explicativos menos simples que el que yo represento. 
suceda (así nunca haya sucedido) es igual al grado de creencia de que A y B sucederán. Quizá aquí nace la tendencia a afirmar que las probabilidades bayesianas miden la incertidumbre. Un ejemplo es la creencia en que "si el cielo está azul entonces no lloverá". Es decir, en ausencia de datos para estimar si es probable que llueva, los eventos relacionados y conocidos (como el cielo azul) nos permite inferir la probabilidad (subjetiva o bayesiana) de lluvia. En este ejemplo, la probabilidad bayesiana no mide la incertidumbre, mide el grado de conocimiento que tenemos sobre un evento, la lluvia. Este grado de conocimiento será mayor si incluimos otros eventos relacionados: temperatura, presiones, etc.

Mi afirmación es que la incertidumbre está referida al desconocimiento de un evento, a la imposibilidad de relacionar este evento desconocido con otros eventos. En el ejemplo anterior sabemos de la posibilidad de lluvia. Nuestras creencias nos permiten estimar, bien o mal, la posibilidad (probabilidad subjetiva o bayesiana) de que llueva o no. Si la probabilidad bayesiana mide incertidumbres, entonces quisiera saber cómo puedo calcular la probabilidad de daño en la salud humana por el uso de transgénicos. Hasta el momento este evento es incierto.

El riesgo, sin necesidad de recurrir a modelos matemáticos o probabilistas, se construye desde la creencia. Es un riesgo cruzar una vía de alto tráfico, nadar en un río turbulento, el contacto con personas infectadas con un virus, transitar una calle desconocida a altas horas de la noche, navegar en Internet sin una protección contra virus. Este riesgo subjetivo sin modelos probabilísticos es el que Kahneman denomina riesgo intuitivo,

The subjective assessment of probability resembles the subjective assessments of physical quantities such as distance or size. These judgments are all based on data of limited validity, which are processed according to heuristic rules.

(Kahneman 2002, 465)

Estas reglas heurísticas basadas en grados de conocimiento bajos, como enuncia Kahneman, son las que han permitido la 
supervivencia del lego en la sociedad del riesgo. El bajo nivel de conocimiento que tiene el lego sobre ciertos estados del mundo, lo obliga a recurrir a estas reglas. Sus decisiones siguen patrones no formales, no incluyen probabilidades objetivas ni subjetivas. Desde sus creencias describen "posibles" estados del mundo, incluyen lo que para Kahneman son las "probabilidades subjetivas"39. Tanto el experto como el lego hacen uso de estas probabilidades subjetivas al evaluar eventos con bajos grados de conocimiento como el resultado de unas elecciones o el de un partido de fútbol.

Sean reglas heurísticas o simples atajos cognitivos, la brecha entre expertos y legos parece estar relegada al grado de conocimiento que uno u otro tiene. Las decisiones del lego y su método de estimación de los riesgos comunes presentes en su vida cotidiana no se pueden solapar a riesgos más complejos, los cuales carecen de la información pública suficiente, o cuyos mecanismos de divulgación han sido débiles.

El riesgo es la creencia anticipada de lo que podría ocurrir en caso de que se llegara a producir la amenaza. El desastre es "la materialización del riesgo". Es decir, lo que era una posibilidad o un grado de creencia, efectivamente sucede. La incertidumbre es ignorancia y, por tanto, no es posible anticipar lo que sucederá, no es posible calcular probabilidad alguna.

\section{CONCLUSIONES}

El significado de palabras como incertidumbre, riesgo y probabilidad no es el mismo entre una disciplina y otra. Este significado varía, incluso, en una misma disciplina. Esta polisemia ha llevado a confundir riesgo con incertidumbre. Lo anterior me ha permitido afirmar que es posible llegar a un consenso en el concepto del riesgo

39 Según Kahneman y Tversky, los juicios intuitivos no son más simples que los modelos racionales. Los juicios intuitivos bajo incertidumbre se basan en la accesibilidad, representatividad y en el anclaje heurístico que constituyen las probabilidades subjetivas o el estimativo (no calculado) de la probabilidad de un evento. Para mayor información sobre los prejuicios en la toma de decisiones bajo incertidumbre, ver (Gilovich, Griffin y Kahneman 2002). 
sólo si se logra el consenso en conceptos como incertidumbre y probabilidad. Es decir, es perentorio utilizar palabras diferentes para conceptos diferentes: posibilidad (likelihood) en lugar de probabilidad subjetiva y, riesgo en lugar de incertidumbre.

Bajo la premisa de una distinción riesgo-incertidumbre, una noción de riesgo plausible con la premisa y lo expuesto en el artículo, es la siguiente: "La posibilidad de un resultado no deseado, el cual es una función de tres factores: el grado de creencia en la pérdida o daño en un sistema (físico, técnico, social, cultural o ecológico) de algo que es valioso (consecuencia), el grado de creencia en la amenaza que es posible que ocasione la pérdida o el daño y, el grado de creencia en la vulnerabilidad del sistema (incluye la creencia o confiablidad en los controles del sistema)".

En torno a la palabra "posibilidad" se acercarán expertos, científicos sociales y el público lego, dado que no se excluyen las probabilidades (con mucha reticencia por parte del lego) y mucho menos las creencias (criticadas por el experto). La "posibilidad" de ocurrencia de un evento sumada a la "posibilidad" de que dicho evento cause pérdida o daño, es suficiente, en mi concepto, para que tanto el lego como el experto hallen el punto de encuentro deseado para la noción de riesgo.

I would like to define the term risk as the possibility that human actions or events lead to consequences that have an impact on what humans value. 


\section{Bibliografía}

Beck, U. (1998 (1986)). La sociedad del riesgo: Hacia una nueva modernidad. (J. Navarro, D. Jiménez, \& B. M. Rosa, Trads.) Barcelona: Paidós.

Beck, U., Giddens, A., \& Lash, S. (1997 (1994)). Modernización reflexiva. Política, tradición y estética en el orden social moderno. Madrid: Alianza Universidad.

Boole. (1854). An investigation on laws of the thought. London.

Carnap, R. (1969 (1966)). Fundamentación lógica de la física. (N. Miguens, Trad.) Buenos Aires: Sudamericana.

Cohen, J., \& Hansel, M. (1956). Risk and Gambling. The Study if Subjective Probability. London: Longmans, Green and Co Ltd.

De Morgan, A. (1847). Formal Logic: or, the Calculus of Inference, necessary and probable. London: Taylor and Walton.

Devey, J. (1854). Logic; or the Science of Inference. London: Henry G. Bohn, York Street, Covent Garden.

Douglas, H. (2000). Inductive Risk and Values in Science. Philosophy of Science, 67 (4), 559-579.

Dubois, D. (2006). Possibility theory and statistical reasoning. Computational Statistics \& Data Analysis, 51, 47-69.

Dubois, D., Prade, H., \& Smets, P. (1996). Representing Partial Ignorance. Trans. on Systems, Man, and Cybernetics, 26, 361-378.

Elster, J. (1990). El cambio tecnológico: investigaciones sobre la racionalidad y la transformación social. Barcelona: Gedisa.

Funtowicz, S. O., \& Ravetz, J. R. (2001). Global risk, uncertainty and ignorance. En J. X. Kasperson, \& R. E. Kasperson (Edits.), Global Environmental Risk. The Unite Nations University Press.

Gilovich, T., Griffin, D., \& Kahneman, D. (Edits.). (2002). Heuristics and Biases: The Psychology of Intuitive Judgment. Cambridge: Cambridge University Press.

Hempel, C. G. (1965). "Science and Human Values" in Aspects of Scientific Explanation. New York: Free Press.

Hempel, C. G. (1979 (1966)). Filosofía de la ciencia natural. Alianza Editorial. 
Kahneman, D. (2002). Maps of Bounded Rationality: a Perspective on Intuitive Judgment and Choise. Recuperado el 2005, de http://nobelprize.org/ nobel_prizes/economics/laureates/2002/kahnemann-lecture.pdf.

Keynes, J. M. (1921). A Treatise on Probability. London: Macmillan and co. limited.

Knight, F. H. (1921). Risk, Uncertainty, and Profit. Boston: Hart, Shaffner \& Marx.

López Cerezo, J. A., \& Luján, J. L. (2000). Ciencia y política del riesgo. Madrid: Alianza Editorial.

Matos, M. A. (2007). Decision under risk as a multicriteria problem. European Journal of Operational Research, 181, 1516-1529.

Peirce, C. S. (1923). Chance, Love and Logic. Philosophicla Essays. (M. Cohen, Ed.) New York: Horcourth, Brace \& Company, INC.

Peirce, C. S. (1877). The Fixation of Belief. Recuperado el 2007, de The Peirce Gateway: http://www.cspeirce.com/menu/library/bycsp/fixation/ fx-frame.htm

Popper, K. (1996 (1990)). Un mundo de propensiones. (J. M. Esteban Cloquell, Trad.) Madrid: Tecnos.

Ramsey, F. P. (1931). Truth and Probability \& "Further Considerations" and "Probability and Partial Belief'. En R. Braithwaite (Ed.), Foundations of Mathematics and other Logical Essays (págs. 156-198). London: Kegan, Paul, Trench, Trubner \& Co. Ltd.

Ravetz, J. (1997). The Science of 'what-if. Futures, 29, 533-539.

Renn, O. (1998). The role of risk perception for risk management. Reliability Engineering and System Safety, 59, 49-62.

Renn, O. (2005). White paper on risk governance: Towards an intergrative approach. Genève: International Risk Governance Council.

Renn, O., \& Rohrmann, B. (Edits.). (2000). Cross-Cultural Risk Perception - A Survey of Empirical Studies. Technology, Risk and Society, 13. Dordrecht: Kluwer Academic Publishers.

Rivadulla, A. (1991). Probabilidad e inferencia científica. Anthropos.

Rosa, E. A. (1998). Metatheoretical Foundations for Post-Normal Risk. Journal of Risk Research, 1, 15-44.

Taroni, F., Garbolino, P., Aitke, C., \& Biederman, A. (2006). Bayesian Networks and Probabilistic Inference in Forensic Science. West Sussex: Willey. 
Venn, J. (1876). The Logic of Chance. London: Macmillan and Co.

von Mises, R. (1981). Probability, Statistics and Truth. New York: Courier Dover Publications.

Wynne, B. (2002). Risk and Environment as Legitimatory Discourses of Technology: Reflexivity Inside Out? Current Sociology, 50 (3), 459-477.

Wynne, B. (1992). Uncertainty and Environmental Learning. Global Environmental Change, 111-127.

Zadeh, L. A. (2005). From search engines to question answering systems. Recuperado el 2007, de http://www.cs.berkeley.edu/ zadeh/

Zadeh, L. A. (1984). Fuzzy Probabilities. Information Processing \& Management, 20 (3), 363-372.

Zadeh, L. A. (1965). Fuzzy Sets. Information and Control, 8, 338-353.

Zadeh, L. A. (2006). Generalized theory o funcertainty (GTU) - principal concepts and ideas. Computational Statistics \& Data Analysis, 51, 15-46.

Zadeh, L. A. (1981). Possibility Theory and Soft Data Analysis. Obtenido de http://www.cs.berkeley.edu/ zadeh/

Zwick, M. M., \& Renn, O. (Edits.). (2002). Perception and Evaluation of Risks. Stuttgart: Center of Technology Assessment. 
